\title{
Optimization of Q-Values for Coherent Optical Transmission Network
}

\author{
Abdul Gafur*1 and M. S. Islam ${ }^{2}$ \\ ${ }^{1}$ Dept. of Electronic and Telecommunications Engineering, IIUC, Chittagong, Bangladesh \\ ${ }^{2}$ Dept. of Physics, Jahangirnagar University, Savar, Bangladesh. \\ *Corresponding Author: abdul.gafur@ete.iiuc.ac.bd
}

\begin{abstract}
In this paper we have analyzed the Gaussian Non-linear Interference $\left(\mathrm{G}_{\mathrm{NLI}}\right)$ spectrum considering non identical channels, non-identical links and Amplified Spontaneous Emission (ASE) noise power spectrum for Coherent Optical Transmission Network (COTN) to calculate the Optical Signal to Noise Ratio (OSNR) and Quality (Q) values. In this study, three different Baud rate values (13.875Gbaud, 27.75Gbaud, and 55.5Gbaud) are considered to compute the Q values and OSNR in the COTN. Consequently, the COTN produces $111 \mathrm{~Gb} / \mathrm{s}, 222 \mathrm{~Gb} / \mathrm{s}$ and $444 \mathrm{~Gb} / \mathrm{s}$ line rates for three different Baud rate values (13.875Gbaud, 27.75Gbaud, and 55.5Gbaud) respectively in PM-16QAM modulation format. It is confirmed that the OSNR is always greater than Q values. It is also found that the differences between OSNR and Q are $0.23 \mathrm{~dB}, 1.73 \mathrm{~dB}$, and $3.24 \mathrm{~dB}$ for $111 \mathrm{~Gb} / \mathrm{s}, 222 \mathrm{~Gb} / \mathrm{s}$ and $444 \mathrm{~Gb} / \mathrm{s}$ line rates respectively. Here transmission of launch power per span, number of channels, number of spans and the fiber dispersion in the optical link are considered.
\end{abstract}

Keywords: Quality values (Q);Optical SNR (OSNR); Erbium Doped Fibre Amplifier (EDFA); Gaussian Non-linear Interference $\left(\mathrm{G}_{\mathrm{NLI}}\right)$

\section{INTRODUCTION (HEADING 1)}

At present higher Baud rate and smaller channel spacing for the super channel are the key factors to optimize and develop the COTN [1-2].Therefore, Q factor is an important parameter to design the high bit rate of optical system network. In this regard, there are reasons to measure the $\mathrm{Q}$ factor for optimizing and developing the existing long haul communication systems which are associated with the six criteria given below [3]. Firstly, Q factor is derived from OSNR; as a result, it is the function of OSNR which measure the performance of receiver section qualitatively for a required SNR. Secondly, we can compute the Q factor scales dynamically because it is related with OSNR. Thirdly, bit error rate calculation is relatively difficult because it takes long time to collect the bit error values for a. fixed target in which system designer follows the worksheet. On the other hand, the fourth reason is that to measure the scale of $Q$ values is reasonably simple because it is performed in $\mathrm{dB}$ format. The fifth criterion is that normally we measure the noise in receiver section using Optical Spectrum Analyzers (OSA). In this case, around $0.0125 \mathrm{THz}$ is the optical bandwidth which is the optical signal measurement scale. In practice, the sixth reason is that the OSNR is greater than the Q scale which varies from $0.2 \mathrm{~dB}$ to $3 \mathrm{~dB}$ where the margin around $2 \mathrm{~dB}$ is at the end of the light detector device.

Consequently, we consider the non-linear propagation effects to measure the $\mathrm{Q}$ factor. Schroedinger equation is the solution to solve the nonlinear model but it takes long time for computation of the system. Nowadays, optical simulator tool is also more expensive 
and simulation process is time consuming [6]. In this respect, GN is the accurate and appropriate model [5], [7], [8], [10] to solve these limitations. In this regard, GN model is used to calculate the PSD of Non Linear Interference (NLI) and also the model reduces the computational complexity for non identical channels and links of the COTN [4], [8].

On the other hand, EDFA is used as a span of the link. Therefore, there is an ASE noise spectrum after each span and amplification is done periodically in the network. Then, we accumulate the ASE noise power and NLI noise power to measure the OSNR after total number of spans. In this way, we follow the optical bandwidth and required electrical bandwidth to measure the $\mathrm{Q}$ values of the optical network.

In this paper, an analytical study is done to generate the PSD of NLI and ASE noises for COTN to determine the OSNR and Q values. It is also shown that the relationship between OSNR and Q for different line rates. Where line rates effect on the COTN are considered to measure the electrical bandwidth. Simulation is performed using MATLAB software to measure the variation between OSNR and Q factor for receiver section in the system. The difference penalty between OSNR and Q factor is simulated considering number of channels, number of spans and input transmitting launch power per span respectively. It is found that the difference between OSNR and Q is $0.2 \mathrm{~dB}$ to $3.2 \mathrm{~dB}$ for $111 \mathrm{~Gb} / \mathrm{s}$ to $444 \mathrm{~Gb} / \mathrm{s}$ data rates considering different system parameters in the COTN.

Model structure for the COTN is in section II. Mathematical expression for quality factor measurement is available in section III. Section V is the simulation results and comments. Finally we put the conclusion in VI.

\section{MODEL STRUCTURE}

The objective of the model of COTN is to measure the OSNR to calculate the Q values considering the ASE and NLI noises in the optical link of the network. In the transmitter section, 11 numbers of channels are used to make a super-channel where $6^{\text {th }}$ channel is the center number of the channels. $f_{c+n}, f_{c-n}$ and $f_{c}$ are the function to make the Even, Odd and Center channel to produce the polarization multiplexing in the transmitter of the COTN. Then modulated optical signal is passed through the optical filter [11]. The optical combiner is used to combine the filtered signal. The Pure Silica Core Fiber [13-14] which is a commercial fiber is used in the link of the optical network to propagate the optical carrier frequency taking the input transmitting power in every span. The specification of the PSCF commercial fiber is presented in Table I. EDFA acts as a span and is repeated after $90 \mathrm{~km}$ optical link in the network. 60 numbers of EDFA are used in this work as a

number of spans. The $\mathrm{p}_{\mathrm{NLI}}$ is the NLI noise power which is measured in the input of the EDFA and the $\mathrm{P}_{\mathrm{ASE}}$ is the ASE noise power calculated after each EDFA in network [12] and is presented in the following Fig.1. [8-9]. After 60 numbers of spans, the ASE and NLI noises are accumulated to The objective of the model of COTN is to measure the OSNR to calculate the Q values considering the ASE and NLI noises in the optical link of the network. In the transmitter section, 11 numbers of channels are used to make a superchannel where $6^{\text {th }}$ channel is the center number of the channels. $f_{c+n}, f_{c-n}$ and $f_{c}$ are the function to make the Even, Odd and Center channel to produce the polarization multiplexing in the transmitter of the COTN. Then modulated optical signal is passed through the optical filter [11].The optical combiner is used to combine the filtered signal. 
The Pure Silica Core Fiber [13-14] which is a commercial fiber is used in the link of the optical network to propagate the optical carrier frequency taking the input transmitting power in every span. The specification of the PSCF commercial fiber is presented in Table I. EDFA acts as a span and is repeated after $90 \mathrm{~km}$ optical link in the network. 60 numbers of EDFA are used in this work as a number of spans. The $\mathrm{p}_{\mathrm{NLI}}$ is the NLI noise power which is measured in the input of the EDFA and the $\mathrm{P}_{\mathrm{ASE}}$ is the ASE noise power calculated after each EDFA in network [12] and is presented in the following Fig. 1. [8-9]. After 60 numbers of spans, the ASE and NLI noises are accumulated to determine the total OSNR. Finally, Q factor is obtained from the OSNR to optimize the COTN.

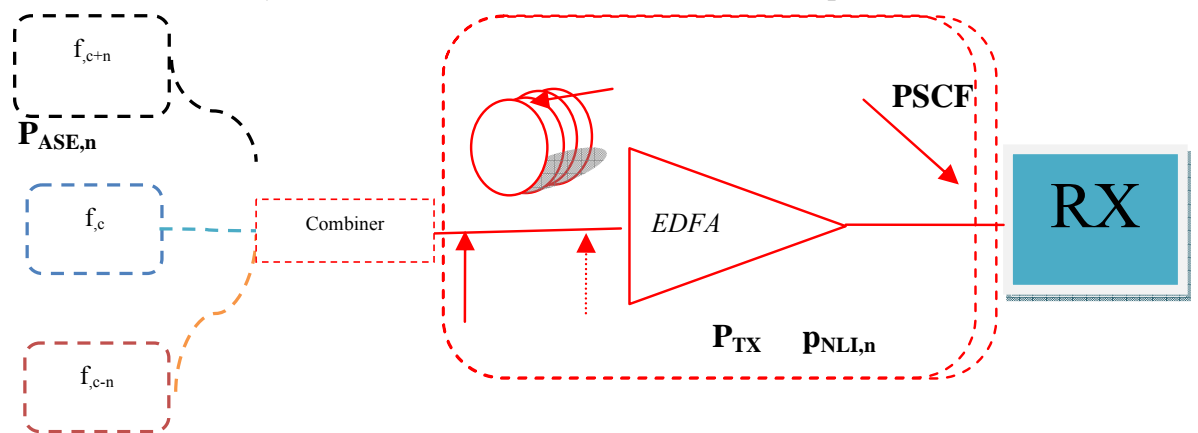

Fig.1. Coherent Optical Transmission Network

TheORETiCal Analysis

To calculate the Power of NLI noise, the Equ. (127)- (129) of [10] is followed and presented below. The factor is that the Gaussian Noise $(\mathrm{GN})$ model is chosen for its non identical channel and non identical link specification of the COTN.

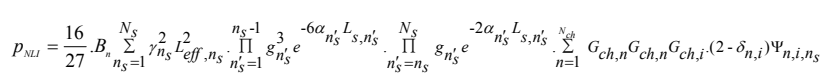

In Equ. (1), $p_{N L I}$ is the power of non linear signal inference of $i^{\text {th }}$ number of the center channel and $N s$ is the total number of optical link in the network. $\gamma$ is the non-linearity coefficient of the PSCF commercial optical fiber and $\mathrm{L}_{\text {eff }}$ is the effective length [12] and presented as follows:

$$
L_{e f f}=\left(1-\exp \left(-\alpha L_{s}\right)\right) / \alpha
$$

Where $\mathrm{L}_{\mathrm{s}}$ is the span length and $\alpha$ is the fiber loss coefficient per kilometer. $g$ is the gain product. $\mathrm{N}_{\mathrm{ch}}$ is the total number of channels. $G_{c h, n}$ and $G_{c h, i}$ are the spectral density for the $n^{\text {th }}$ and $i^{\text {th }}$ channel in the transmitter part [16] like below:

$$
\begin{aligned}
G_{c h, n} & =\frac{P_{c h, n}}{R_{s}} \\
G_{c h, i} & =\frac{P_{c h, i}}{R_{s}}
\end{aligned}
$$


In Equ. (3) and Equ.(4) $P_{c h, n}$ and $P_{c h, i}$ are the channel power for $n^{\text {th }}$ and $i^{\text {th }}$ channels. $R_{s}$ is the line rate of the channel. In this work three different types of line rates [19] are presented to measure the $\mathrm{Q}$ factor.

$\delta_{n, i}$ and $\Psi_{n, i, n_{s}}$ are the operators to choose the $n^{\text {th }}$ and $i^{\text {th }}$ channel to generate the PSD using GN model. When $n=i$ and $n \neq i$, the $\Psi_{n, i, n_{s}}$ and $\Psi_{i, i, n_{s}}$ work respectively in the following way [10]:

$$
\begin{aligned}
\Psi_{n, i, n_{s}}= & \frac{\operatorname{asinh}\left(\pi^{2}\left(2 \alpha_{n_{s}}\right)^{-1}\left|\beta_{2, n_{s}}\right|\left[f_{c h, n}-f_{c h, i}+B_{c h, n} / 2\right] B_{c h, i}\right)}{4 \pi\left(2 \alpha_{n_{s}}\right)^{-1}\left|\beta_{2, n_{s}}\right|} \\
& -\frac{\left.\operatorname{asinh}\left(\pi^{2}\left(2 \alpha_{n_{s}}\right)^{-1}\left|\beta_{2, n_{s}}\right| f_{c h, n}-f_{c h, i}-B_{c h, n} / 2\right] B_{c h, i}\right)}{4 \pi\left(2 \alpha_{n_{s}}\right)^{-1}\left|\beta_{2, n_{s}}\right|} \\
\Psi_{i, i, n_{s}}= & \frac{\operatorname{asinh}\left(\frac{\pi^{2}}{2}\left|\beta_{2, n_{s}}\right|\left[2 \alpha_{n_{s}}\right]^{-1} B_{c h, i}^{2}\right)}{2 \pi\left|\beta_{2, n_{s}}\right|\left[2 \alpha_{n_{s}}\right]^{-1}}
\end{aligned}
$$

$B_{c h}$ and $\beta_{2}$ are the bandwidth of the channel and disperion[15] of the fiber is used in equ.(5) and (6). asinh controls the array of complex number [17]. ASE noise spectral density is used [18] as below:

$$
G_{A S E}=F h f_{o} A_{S}
$$

In Equ.(7), $F, h, f_{o}$ and $A_{s}$ are the noise figures, plank constant, center frequency and loss of the link. Loss of the link depends on fiber loss per kilometer and length of the link. To measure the Q factor, the OSNR [18] is required.

$$
\text { OSNR }=\frac{P_{T X}}{N_{s}\left(G_{A S E}+G_{N L I}\right) B_{n}}=\frac{P_{T X}}{N_{s}\left(P_{A S E}+p_{N L I}\right)}
$$

Where $B_{n}$ is optical bandwidth which is multiplied with $G_{A S E}$ and $G_{N L I}$ to obtain the $P_{A S E}$ and $p_{N L I}$ respectively. Then, scaling the transmitting input power per link is used to measure the optimum OSNR and Q factor of the network [5].

$$
\text { OSNR }=\frac{P_{T X}}{\left(P_{A S E}+P_{T X}^{3} p_{N L I} B_{c h}^{-3}\right)}
$$

The Q factor is derived from OSNR and is used as an Equ.(10) in [10] and Equ.(4-11) in [3] as given below:

$$
Q=20 \log \sqrt{O S N R} \sqrt{\frac{B_{n}}{R_{s}}}
$$

\section{SIMULATION AND RESULTS}

In this section Matlab programming is run to measure the $\mathrm{Q}$ values of the COTN. As a result, theoretical model used in section III for NLI and ASE are simulated according to the given data in Table I and Table II. Consequently, Table III represents the output of the 
simulation. In this case, modulation format PM-16QAM is used to calculate bit rate $\left(R_{B}\right)$ of the system. This format transfers eight bit per symbol. In this simulation, three different types of Baud rate [19] are used to observe the quality of the network. The line rate of the channel $13.875 \mathrm{Gbaud}, 27.75 \mathrm{Gbaud}$ and $55.5 \mathrm{Gbaud}$ produces the $111 \mathrm{Gbit} / \mathrm{s}$, $222 \mathrm{Gbit} / \mathrm{s}$ and $444 \mathrm{Gbit} / \mathrm{s}$ bit rate respectively for PM-16QAM. This work confirms the required quality of the system for these three different types of Baud rate with assumed parameters.

TABLE I.

SPECIFICATION OF PSCF [13] -[14]
\begin{tabular}{|c|c|c|c|}
\hline Fiber Types & $\alpha[\mathrm{dB} / \mathrm{km}]$ & $\gamma[1 / \mathrm{W} / \mathrm{km}]$ & $\mathrm{D}[\mathrm{ps} / \mathrm{nm} / \mathrm{km}]$ \\
\hline PSCF & 0.162 & 0.60 & 21 \\
\hline
\end{tabular}

TABLE II.

Simulation PARAMETERS
\begin{tabular}{|c|c|}
\hline Parameters & Data \\
\hline Channels $\left(N_{c h}\right)$ & 11 \\
\hline Operating wavelength $(\lambda)$ & $1550[\mathrm{~nm}]$ \\
\hline Channel Spacing $(\Delta f)$ & $0.05[\mathrm{THz}]$ \\
\hline Span Length $(L s)$ & $90[\mathrm{~km}]$ \\
\hline Number of span $(N s)$ & 60 \\
\hline Noise Figure $(F)$ & $6[\mathrm{~dB}]$ \\
\hline Center frequency $\left(f_{c}\right)$ & $193.41[\mathrm{THz}]$ \\
\hline Channel Bandwidth $\left(B_{c h}\right)$ & $0.032[\mathrm{THz}]$ \\
\hline Optical Noise Bandwidth $\left(B_{n}\right)$ & $0.0125[\mathrm{THz}]$ \\
\hline
\end{tabular}

TABLE III. SimUlation DATA FOR REQUiRED Q VAlUES

\begin{tabular}{|c|c|c|c|}
\hline $\boldsymbol{R}_{\boldsymbol{s}}[\mathbf{G b a u d}]$ & $\boldsymbol{B}_{\boldsymbol{n}}[\mathbf{T H z}]$ & $\boldsymbol{R}_{\boldsymbol{B}}[\mathbf{G b i t} / \mathbf{s}]$ & $\boldsymbol{O S N R}[\mathbf{d B}]-Q[\boldsymbol{d B}]$ \\
\hline 13.875 & 0.0125 & 111 & 0.23 \\
\hline 27.75 & 0.0125 & 222 & 1.73 \\
\hline 55.5 & 0.0125 & 444 & 3.24 \\
\hline
\end{tabular}

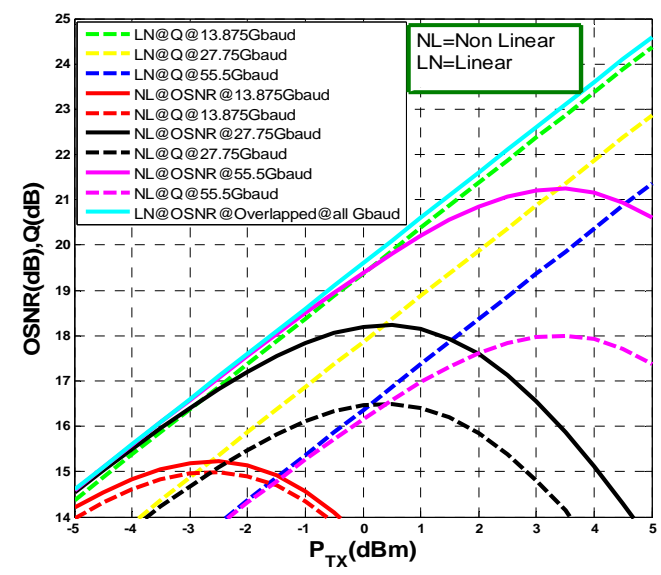

Fig.2. Q (dB), OSNR (dB) vs input launch power per span $\mathrm{P}_{\mathrm{TX}}(\mathrm{dBm})$ for three different Baud rates. 
Fig.2 presents the OSNR (dB)and Q (dB) versus input transmitting power per span $\left(\mathrm{P}_{\mathrm{TX}}\right)$ $[\mathrm{dBm}]$.In the results, the OSNR and $\mathrm{Q}$ values for linear and non linear propagations are measured. The solid line is for OSNR performance of the system where dotted lines convey the information for $\mathrm{Q}$ values. The red, black and magenta color lines are for 13.875 Gbaud rate, 27.75Gbaud and 55.5 Gbaud symbol rates for non linear propagation respectively. The dotted blue, yellow and green color lines show the linear propagation. For linear propagation, we consider only ASE noise power where non linear interference $\left(\mathrm{p}_{\mathrm{NLI}}\right)$ power is off during linear propagation. In the case of OSNR, the results get overlapped because of linear propagation which is denoted by Cyan color line. It is also found that in optimum point values, the linear and non linear difference is $1.76 \mathrm{~dB}$. It is also found that, for linear confirmation, the $\mathrm{P}_{\mathrm{TX}}$ is changed along with OSNR by $1 \mathrm{~dB}$ linearly. Here, considering 13.875 Gbaud rate, the optimum $\mathrm{P}_{\mathrm{TX}}$ is $-2.5 \mathrm{dBm}$ when the optimum OSNR and Q are $15.23 \mathrm{~dB}$ and $14.99 \mathrm{~dB}$ respectively. In this rate, the difference between OSNR and $\mathrm{Q}$ values is $0.23 \mathrm{~dB}$.For 27.75 Gbaud rates, the optimum $\mathrm{P}_{\mathrm{TX}}$ is $0.5 \mathrm{dBm}$ when the optimum OSNR and $\mathrm{Q}$ are $18.23 \mathrm{~dB}$ and $16.49 \mathrm{~dB}$ respectively. Consequently, the difference between the OSNR and Q values is 1.73dB.For 55.5 Gbaud rates, the optimum $\mathrm{P}_{\mathrm{TX}}$ is $3.5 \mathrm{dBm}$ when the optimum OSNR and $\mathrm{Q}$ are $21.24 \mathrm{~dB}$ and $18.00 \mathrm{~dB}$ respectively. As a result,the difference between the OSNR and Q values is $3.24 \mathrm{~dB}$.

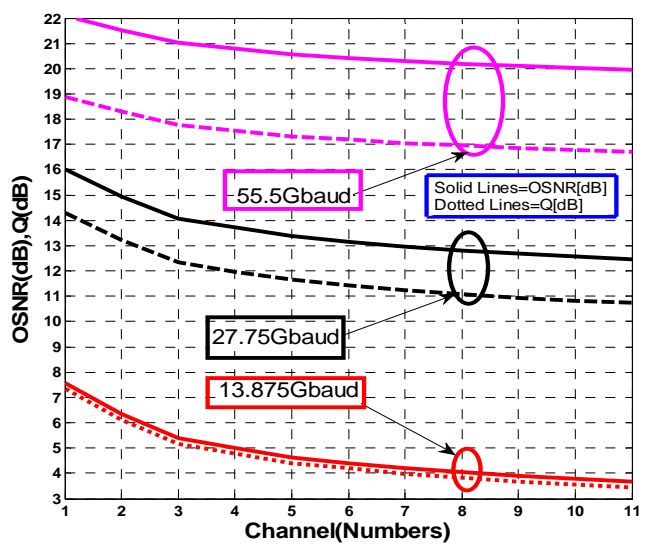

Fig.3. Q (dB), OSNR (dB) vs Channel Numbers with different values of symbol rates

Fig.3. presents the channel numbers versus $Q(\mathrm{~dB})$ and OSNR $(\mathrm{dB})$ in the COTN. The solid and dotted lines represent the OSNR and Q values respectively. The red, black and Magenta colour lines are for $13.875 \mathrm{Gbaud}, 27.75 \mathrm{Gbaud}$ and $55.5 \mathrm{Gbaud}$ respectively. The OSNR is going down with the addition of channels in the network. The result shows that, for 13.875 Gbaud symbol rate, the end of $Q$ value and OSNR is $3.44 \mathrm{~dB}$ and $3.66 \mathrm{~dB}$ respectively. Where the difference between OSNR and Q is $0.23 \mathrm{~dB}$. The OSNR(end) and $Q$ (end) for $11^{\text {th }}$ number of channel are $12.46 \mathrm{~dB}$ and $10.73 \mathrm{~dB}$ using $27.75 \mathrm{Gbaud}$ symbol rate. As a result, $1.73 \mathrm{~dB}$ is the difference between OSNR and $\mathrm{Q}$ values. At $11^{\text {th }}$ number of channel position, the OSNR and Q are $19.95 \mathrm{~dB}$ and $16.71 \mathrm{~dB}$ respectively considering $55.5 \mathrm{Gbaud}$ symbol rate. Thus the difference between OSNR and Q is 3.24dB. 


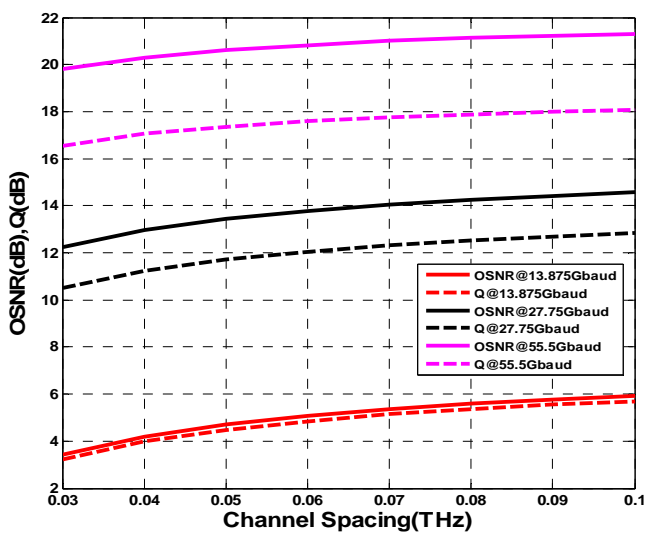

Fig4. Q (dB), OSNR (dB) vs Channel Spacing (THz) with different values of symbol rates considering PSCF

Fig.4. shows the channel spacing effects with respect to the $\mathrm{Q}(\mathrm{dB})$ and OSNR $(\mathrm{dB})$ in the network. The dotted and solid lines present the $\mathrm{Q}$ values and OSNR respectively. Red, black and magenta colour lines bear the 13.875Gbaud, 27.75Gbaud and 55.5Gbaud symbol rates respectively. The OSNR and Q values are going up with the increment values of channel spacing in network. The ASE and NLI noise effects are considered to simulate the OSNR and Q factor. For the value of $0.1 \mathrm{THz}$ channel spacing, it is found that the $\mathrm{Q}$ factor is around $5.69 \mathrm{~dB}, 12.82 \mathrm{~dB}$ and $18.06 \mathrm{~dB}$ where OSNR is approximately 5.92 $\mathrm{dB}, 14.55 \mathrm{~dB}$ and $21.30 \mathrm{~dB}$ for $13.875 \mathrm{Gbaud}, 27.75 \mathrm{Gbaud}$ and $55.5 \mathrm{Gbaud}$ symbol rates respectively. It is observed that $\mathrm{Q}$ values are less than OSNR by $0.23 \mathrm{~dB}, 1.73 \mathrm{~dB}$ and3.24dB for $13.875 \mathrm{Gaud}, 27.75 \mathrm{Gbaud}$ and $55.5 \mathrm{Gbaud}$ symbol rates respectively.

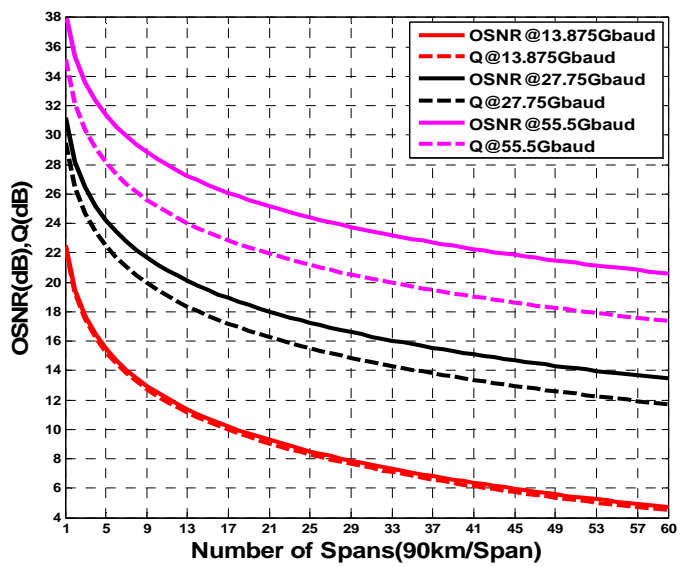

Fig 5. Q (dB), OSNR (dB) vs number of span $(90 \mathrm{~km} / \mathrm{span})$ with different values of symbol rates considering PSCF. 
Fig.5is the performance curve of OSNR $(\mathrm{dB})$ and $\mathrm{Q}(\mathrm{dB})$ versus total number of spans where $90 \mathrm{~km}$ is per span length. Thus, $5600 \mathrm{~km}$ is the total link distance. In the results, red, black and magenta color lines convey the information for $13.875 \mathrm{Gbaud}, 27.75 \mathrm{Gbaud}$ and 55.5Gbaud symbol rates respectively. The solid and dotted lines represent the OSNR and Q performance in the graph. The OSNR and Q performance curves go down when the number of span is added in the network. For $13.875 \mathrm{Gbaud}$ symbol rate, the OSNR (end) and $\mathrm{Q}$ (end) are $4.71 \mathrm{~dB}$ and $4.49 \mathrm{~dB}$ respectively. In the figure, when symbol rate is 27.75Gbaud, the OSNR (end) and Q (end) are $13.4416 \mathrm{~dB}$ and $11.71 \mathrm{~dB}$. It is found that 55.5Gbaud makes $20.61 \mathrm{~dB}$ OSNR (end) and $17.37 \mathrm{~dB} \mathrm{Q}$ (end) in the network. In this performance, it is observed that, $111 \mathrm{~Gb} / \mathrm{s}, 222 \mathrm{~Gb} / \mathrm{s}$ and $444 \mathrm{~Gb} / \mathrm{s}$ bit rates show the difference between OSNR and $\mathrm{Q}$ as $0.23 \mathrm{~dB}, 1.73 \mathrm{~dB}$ and $3.24 \mathrm{~dB}$ respectively.

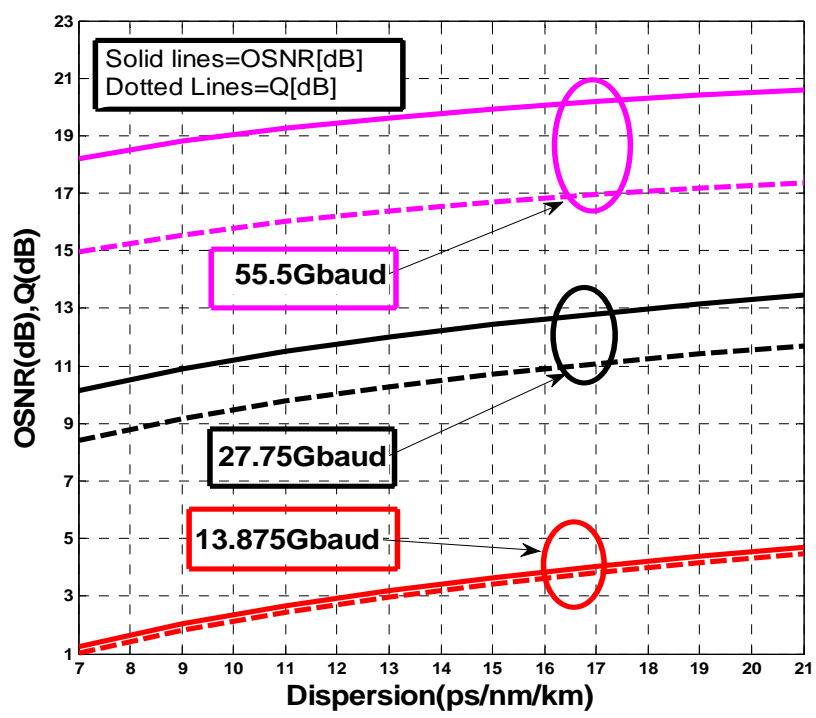

Fig. 6. Q (dB), OSNR (dB) vs fiber dispersion coefficient $(\mathrm{ps} / \mathrm{nm} / \mathrm{km})$ with different values of symbol rates.

Fig.6 is the performance curve for fiber dispersion $(\mathrm{ps} / \mathrm{nm} / \mathrm{km})$ versus OSNR $(\mathrm{dB})$ and $\mathrm{Q}(\mathrm{dB})$ considering $111 \mathrm{~Gb} / \mathrm{s}, 222 \mathrm{~Gb} / \mathrm{s}$ and $444 \mathrm{~Gb} / \mathrm{s}$ bit rates. In this performance, it is observed that the OSNR is going up with the increment of dispersion values in the optical link. In this figure, magenta, black and red color lines are the $13.875 \mathrm{Gbaud}, 27.75 \mathrm{Gbaud}$ and 55.5Gbaud symbol rates. Solid and dotted lines are the OSNR and Q values in the curve. For dispersion $21(\mathrm{ps} / \mathrm{nm} / \mathrm{km})$, the OSNR and $\mathrm{Q}$ are $4.71 \mathrm{~dB}$ and $4.48 \mathrm{~dB}$ when symbol rate is $13.875 \mathrm{Gbaud}$.For $27.75 \mathrm{Gbaud}$, the OSNR and Q are $13.44 \mathrm{~dB}$ and $11.7 \mathrm{~dB}$. $20.6 \mathrm{~dB}$ and $17.37 \mathrm{~dB}$ are the OSNR and $\mathrm{Q}$ values considering the $55.5 \mathrm{Gbaud}$ symbol rate. It is observed that the differences between OSNR and Q are $0.23 \mathrm{~dB}, 1.73 \mathrm{~dB}$ and $3.24 \mathrm{~dB}$ for $111 \mathrm{~Gb} / \mathrm{s}, 222 \mathrm{~Gb} / \mathrm{s}$ and $444 \mathrm{~Gb} / \mathrm{s}$ line rates respectively. 


\section{Conclusion}

In this paper we have analyzed the Q values considering NLI and ASE noise effects on the COTN for three different symbol rates with commercially available PSCF optical fiber. It is observed that the difference between OSNR and Q varies from $0.23 \mathrm{~dB}$ to $3 \mathrm{~dB}$ for three different symbol rates. It is also stated that for low value of data rate, many channels are required where many transmitters and receivers are involved to get the target capacity which makes the system large and complex. Therefore, the system needs many channels and more input transmitting launch power per span. Thus, for a demanded Q value, an optimum baud rate is preferred to design the COTN.

\section{References}

[1] V. Bobrovs, A. Udalcovs, R. Parts, and I. Trifonovs, "Evaluation of Nonlinear Effect Impact on Optical Signal Transmission over Combined WDM System," PIERS Proceedings, Taipei, Mar. 2013.

[2] Abdul Gafur and M. S. Islam, "Quality Factor of Coherent Optical Transmission Systems with Different Baud Rates,” ICMEIE Proceedings, 05-06 June, 2015, Rajshahi, Bangladesh.

[3] Tony Antony, Ashwin Gumaste, "WDM Network Design”, CiscoPress, Feb.2003; http://www. ciscopress. com/articles/article. $a s p ? p=30886 \&$ seqNum $=4$

[4] Pontus Johannisson and Erik Agrell, "Modeling of Nonlinear Signal Distortion in FiberOptical Networks", posted on arXiv, www.arxiv.org, paper identifier: 1309.4000v1, 16 sep. 2013.

[5] Pierluigi Poggiolini, "The GN Model of Non-Linear Propagation in Uncompensated Coherent Optical Systems,” J. Lightw. Technol., vol. 30, no.24, Dec. 2012.

[6] Kerry Hinton, J. C. Li,Peter M. Farrel, Wayne V. Sorin, "A New Design Technique for Optical Links", WU1, 15.30 - 15.45, 2009.

[7] P. Poggiolini, A. Carena, V. Curri, G. Bosco, and F. Forghieri, "Analytical modeling of nonlinear propagation in uncompensated optical transmission links," IEEE Photon. Technol. Lett., vol. 23, no. 11, pp. 742-744, Jun. 2011.

[8] A. Carena, V. Curri, G. Bosco, P. Poggiolini, and F. Forghieri, "Modeling of the impact of nonlinear propagation effects in uncompensated optical coherent transmission links," J. Lightw. Technol., vol. 30, no. 10, pp. 1524-1539, May 2012.

[9] Abdul Gafur, Md. Razu Ahmed, Md. Badiuzzaman and Abu Riduan Md Foisal, "OSNR Improvement of Coherent Uncompensated Optical Transmission Systems for Various Commercial Optical Fiber Types," ICEEICT Proceedings, 10-12 April, 2014, Dhaka, Bangladesh.

[10] Pierluigi Poggiolini, Gabriella Bosco, Andrea Carena, Vittorio Curri, Yanchao Jiang and Fabrizio Forghieri, "A Detailed Analytical Derivation of the GN Model of Non-Linear Interference in Coherent Optical Transmission Systems", posted on arXiv, www.arxiv.org, paper identifier :1209.0394, (2012).

[11] A. Carena, G. Bosco, V. Curri, P. Poggiolini, F. Forghieri, "Impact of the Transmitted Signal Initial Dispersion Transient on the Accuracy of the GN-Model of Non-Linear Propagation", Eur. Conf. Opt. Commun. (ECOC), paper Th.1.D.4, 2013. 
[12] P. Poggiolini, G. Bosco, A. Carena, R. Cigliutti, V. Curri, F. Forghieri, R. Pastorelli, S. Piciaccia, "The LOGON Strategy for Low-Complexity Control Plane Implementation in NewGeneration Flexible Networks," Opt. Fiber Commun. Conf. (OFC/NFOEC), OSA, 2013.

[13] A. Nespola, S. Straullu, A. Carena, G. Bosco, R. Cigliutti, V. Curri, P. Poggiolini, M. Hirano, Y. Yamamoto, T. Sasaki, J. Bauwelinck, K. Verheyen, F. Forghieri, "Extensive Fiber Comparison and GN-model Validation in Uncompensated Links using DAC-generated Nyquist-WDM PM-16QAM Channels," Opt. Fiber Commun. Conf. (OFC/NFOEC) Technical Digest, OSA, 2013.

[14] Vittorio Curri, Pierluigi Poggiolini, Gabriella Bosco, Andrea Carena, and Fabrizio Forghieri, "Performance Evaluation of Long-Haul $111 \mathrm{~Gb} / \mathrm{s}$ PM-QPSK Transmission Over Different Fiber Types," IEEE Photon. Technol. Lett., vol. 22, no. 19, Oct. 2010.

[15] G. P. Agrawal, Fiber-Optic Communication Systems, $4^{\text {th }}$ ed. John Wiley \& Sons, 2010.

[16] G. Bosco, A. Carena, R. Cigliutti, V. Curri, P. Poggiolini, "Next generation Terabit Optical networks: theory, simulation and experiments," Poster-session, DET-day, http://www. det.polito. it/focus/det_day/session_1_14_15_15_00.

[17] URL:http://www.mathworks.it/it/help/matlab/ref/asinh.html

[18] Vittorio Curri, Andrea Carena, Pierluigi Poggiolini, Gabriella Bosco, and Fabrizio Forghieri, "Extension and validation of the GN model for non-linear interference to uncompensated links using Raman amplification," Optical Express, Vol. 21, No. 3,11, Febr

[19] Uary 2013.

[20] Pierluigi Poggiolini, Gabriella Bosco, Andrea Carena, Vittorio Curri, Valerio Miot, and Fabrizio Forghieri, "Performance Dependence on Channel Baud-Rate of PM-QPSK Systems Over Uncompensated Links,” IEEE Photon. Technol. Lett., VOL. 23, No. 1, January 1, 2011 